\title{
Calcium Influx Blockade as Cancer Therapy
}

\section{James L. Cox*}

Department of Biochemistry, A.T. Still University, 800 West Jefferson Street Kirksville, MO 63501, USA

Metastasis is the major reason for treatment failure when it comes to therapy for different cancers. Here I would like to give a brief overview of some research that has attempted to block metastasis through inhibition of calcium signaling and metabolism. This strategy to inhibit metastasis through blocking calcium channels/calcium function is not yet being applied clinically, but could be within several years following clinical trials. It remains an open question whether this calcium strategy will lead to durable improvements for the management of cancer. A number of recent advances in understanding calcium regulation in cancer cells has also brought us closer to possible clinical application of this strategy, as new calcium interfering drugs are being developed based on new molecular and cellular physiological information.

A large amount of research has gone into describing altered expression of calcium channels for a variety of different cancers [1]. Here it will not be possible to cover the details of these findings, but suffice it to say, the selectivity of inhibitors would generally be through targeting the calcium channel type displaying increased expression. It is generally believed the increased expression of a specific calcium channel contributes to a more invasive phenotype for a given cancer. Once a particular overexpressed calcium channel has been identified for a patient's cancer, then an agent directed against that particular channel could be added into the standard therapeutic combination of drugs. Properly applied, a calcium channel inhibition strategy could result in long term benefit and even perhaps longer patient survival.

One major advantage of blocking calcium channels to block cell migration is that there appears to be a universal requirement for calcium membrane transients for cell migration. The idea is that if one could selectively block cancer cell migration this would inhibit cancer cell spread and produce a cytostatic effect that may be synergistic when added to other current anti-cancer therapies. A major hurdle would be finding enough selectivity for cancer cells and of course minimizing any other side effects encountered with these calcium blocking agents. To that end, periodic treatment with calcium interfering agents may have tolerable profiles if targeting can be restricted to those calcium systems which display altered expression in the cancer. Research arising in the past few years has given us examples of different key calcium transport channels which are up-regulated in different cancers [2]. The excellent work with the Ori-Stim calcium regulation system and breast cancer cell migration has begun to suggest the potential application here of new agents to cancer cell migration [3]. Another family of calcium channels that play a prominent role in cancer cell migration is the TRPC (Transient Receptor Potential Canonical) family [4]. Together these two channel types of calcium regulation will provide new targets for interference with metastasis for a number of common cancer types.

The involvement of calcium transients in cell migration has been known for some time [5]. Whereas certain normal cells, including fibroblasts, use high voltage activated (HVA) type calcium channels, most transformed cells rely on Low Voltage Activated (LVA) and Non-Voltage-Gated (NVG) $\mathrm{Ca}^{+2}$ influx channels [6]. Earlier work by our laboratory showed ionic gadolinium is a potent inhibitor of cancer cell migration [7]. The blockade of calcium influx with $\mathrm{Gd}^{+3}$ or $\mathrm{Tb}^{+3}$ inhibited melanoma cell migration and enhanced cell adhesion. Work by Huang et al. also showed the blockade of calcium transients and cell migration with low $\mu \mathrm{M}$ gadolinium for HT1080 fibrosarcoma cells [8]. Further development of the lanthanides to cancer treatment must await tumor targeted delivery methods to circumvent toxicity issues.

Continuing work on the calcium channel blocker CAI (Carboxyamidotriazole) has paved the way for the calcium channels blocker type of strategy because it has undergone clinical testing in late stage cancer patients with some disease stabilization [9]. This small molecule calcium channel inhibitor blocks cancer cell migration by targeting non-voltage gated calcium channels. Since CAI is a reversible inhibitor there would need to be a constant application to achieve cytostatic effects. One nice advantage, however, is that it may also block angiogenesis [10]. Of foremost importance for CAI is the favorable pharmacokinetic profile and apparent lack of serious side effects.

As new details emerge for the regulation of cancer cell migration by calcium transport mechanisms, new therapeutic possibilities will undoubtedly be realized. Altogether, this strategy is an untapped, potential brake for metastatic cells which may go a long way towards potentially improving cancer therapy. In addition, calcium has other important roles such as in cell replication and apoptosis which certainly also would have application to cancer treatment through new strategies of blocking or induction, respectively. Advances for cancer therapy concerning these other calcium dependent functions are, however, a tale for another time.

\section{References}

1. Monteith GR, McAndrew D, Faddy HM, Roberts-Thomson SJ (2007) Calcium and cancer: targeting Ca2+ transport. Nat Rev Cancer 7: 519-530.

2. Cuddapah VA, Sontheimer H (2011) lon channels and transporters [corrected] in cancer. 2. Ion channels and the control of cancer cell migration. Am J Physiol Cell Physiol. Cell physiology 301: 541-549.

3. Yang S, Zhang JJ, Huang XY (2009) Orai1 and STIM1 are critical for breast tumor cell migration and metastasis. Cancer cell 15: 124-134.

4. Bomben VC, Turner KL, Barclay TT, Sontheimer H (2011) Transient receptor potential canonical channels are essential for chemotactic migration of human malignant gliomas. J Cell Physiol 226: 1879-1888.

5. Brundage RA, Fogarty KE, Tuft RA, Fay FS (1991) Calcium gradients

*Corresponding author: James L. Cox, Department of Biochemistry, A.T. Still University, 800 West Jefferson Street Kirksville, MO 63501, USA, E-mail: jcox@atsu.edu

Received October 31, 2012; Accepted October 31, 2012; Published November 05, 2012

Citation: Cox JL (2012) Calcium Influx Blockade as Cancer Therapy. J Carcinogene Mutagene 3:e107. doi:10.4172/2157-2518.1000e107

Copyright: (c) 2012 Cox JL. This is an open-access article distributed under the terms of the Creative Commons Attribution License, which permits unrestricted use, distribution, and reproduction in any medium, provided the original author and source are credited. 
underlying polarization and chemotaxis of eosinophils. Science 254: 703-706.

6. Schwab A, Fabian A, Hanley PJ, Stock C (2012) Role of ion channels and transporters in cell migration. Physiol rev 92: 1865-1913.

7. Cox JL, Lancaster T, Carlson CG (2002) Changes in the motility of B16F10 melanoma cells induced by alterations in resting calcium influx. Melanoma Res 12: $211-219$

8. Huang JB, Kindzelskii AL, Clark AJ, Petty HR (2004) Identification of channels promoting calcium spikes and waves in HT1080 tumor cells: their apparent roles in cell motility and invasion. Cancer Res 64: 2482-2489.

9. Azad N, Perroy A, Gardner E, Imamura CK, Graves C, et al. (2009) A phase I study of paclitaxel and continuous daily CAI in patients with refractory solid tumors. Cancer Biol Ther 8: 1800-1805.

10. Oliver VK, Patton AM, Desai S, Lorang D, Libutti SK, et al. (2003) Regulation of the pro-angiogenic microenvironment by carboxyamido-triazole. J Cell Physiol 197: $139-148$ 